\title{
Gynecomastia Surgery Is Associated with Improved Nipple Location in Young Korean Patients
}

\author{
Bo Hyung Lee, Yu Jin Kwon, Jang Wan Park, Jae Ha Hwang, Kwang Seog Kim, Sam Yong Lee \\ Department of Plastic and Reconstructive Surgery, Chonnam National University Medical School, Gwangju, Korea
}

Background Gynecomastia is benign enlargement of breast tissue in males and is fairly common. Mastectomy not only helps in improving the shape of anterior chest, but can also improve the location of nipple. Therefore, a principle element of mastectomy design is defining the normal location of nipple based on major anatomical reference points. Here, the nipple location was compared for before and after gynecomastia surgery. In addition, the same was also compared between male patients undergoing gynecomastia surgery and control group of subjects without gynecomastia.

Methods We retrospectively analyzed gynecomastia patients who underwent conventional subcutaneous mastectomy. Preoperative and postoperative anatomical landmark distances and chest circumferences were measured and compared to the same anthropometric data from 20 healthy adult male controls.

Results Nipple locations were compared among 13 patients and 20 controls. The mean weight of resected breast tissue was $246 \mathrm{~g}$, and overall patient satisfaction grade was 4.3 out of 5 . In the patient group, the slopes for the height-distance from the sternal notch to the nipple and chest circumference-distance between the mid-line of the sternum and the nipple were 0.175 and 0.125 postoperatively, respectively. The slopes of the control group were 0.122 and 0.177 , respectively; these differences were statistically significant $(P<0.05)$.

Conclusions Nipple positions were considerably lower in patients with gynecomastia than in control subjects. Subcutaneous mastectomy was associated with mild elevations, but postoperative locations were still lower compared to controls. Further efforts are needed to improve the location of postoperative nipple-areola complex in patients with gynecomastia.

Keywords Gynecomastia / Male / Breast / Mastectomy / Nipples
Correspondence: Sam Yong Lee Department of Plastic and Reconstructive Surgery, Chonnam National University Medical School, 42 Jebong-ro, Dong-gu, Gwangju 501-757, Korea Tel: +82-62-220-6363 Fax: +82-62-227-1639 E-mail: sylee@chonnam.ac.kr

No potential conflict of interest relevant to this article was reported.

\section{INTRODUCTION}

Gynecomastia refers to the feminine breast development in men and is the most frequent breast condition found among male patients. It can be caused by a variety of factors, including mammary gland tissue hypertrophy, subcutaneous fat accumulation, hormone imbalance, etc. [1].

Several treatment options are available for gynecomastia, including subcutaneous mastectomy, ultrasound liposuction, and endoscopic mastectomy [2]. Satisfaction after gynecomastia re- 
duction treatments depend upon the new breast shape and size as well as the location of nipple-areola complex (NAC). While the postoperative NAC location has been studied extensively, most of the available data had been collected from female populations and cannot be generalized to male patients. While few studies are available for male patients undergoing gynecomastia treatment, NAC have not been sufficiently compared between preoperative and postoperative states.

In this study, we investigated NAC positions before and after gynecomastia treatment in Korean patients and compared the postoperative NAC position to a control group of Korean adults without gynecomastia.

\section{METHODS}

\section{Participants}

A retrospective case-control study was performed. The patient group consisted of 13 patients who underwent subcutaneous mastectomy and liposuction between January 2007 and September 2013. The majority of these patients had bilateral gynecomastia, with a single patient having had unilateral gynecomastia. The control group was recruited through a colleague, and comprised of 20 healthy Korean males with no current or past history of gynecomastia, congenital thoracic malformations, altered thorax, or thoracic operations. All of the operations in the case group were performed by a single surgeon.

\section{Preoperative evaluation}

Preoperative ultrasonography was performed in all patients to identify the main component of gynecomastia. In most cases, either fat or glandular tissue accounted for the volume. In each patient, the surgical option that best addressed the composition of gynecomastia was offered: either subcutaneous mastectomy alone or subcutaneous mastectomy with liposuction.

Anthropometry was obtained for breast height, weight, chest circumference, distance from nipple center to sternal notch, distance from sternal notch to the xiphoid process, distance from sternal notch to nipple, and distance from acromioclavicular joint to nipple. Chest circumferences were measured at the nipple level. These measurements were obtained twice for the patient group (before and after operation) and once for the control group. At each of the measurement session, standard upright position photographs were obtained. All of the measurements were obtained by a single researcher to eliminate intraobserver variability.

Patient satisfaction levels were graded as follows: 1) high dissatisfaction; 2) low dissatisfaction; 3) average; 4) low satisfaction; and 5) high satisfaction.

\section{Fig. 1. Anatomical landmarks}

SN, sternal notch; N, nipple; ACJ, acromioclavicular joint; Sm, sternal midline.

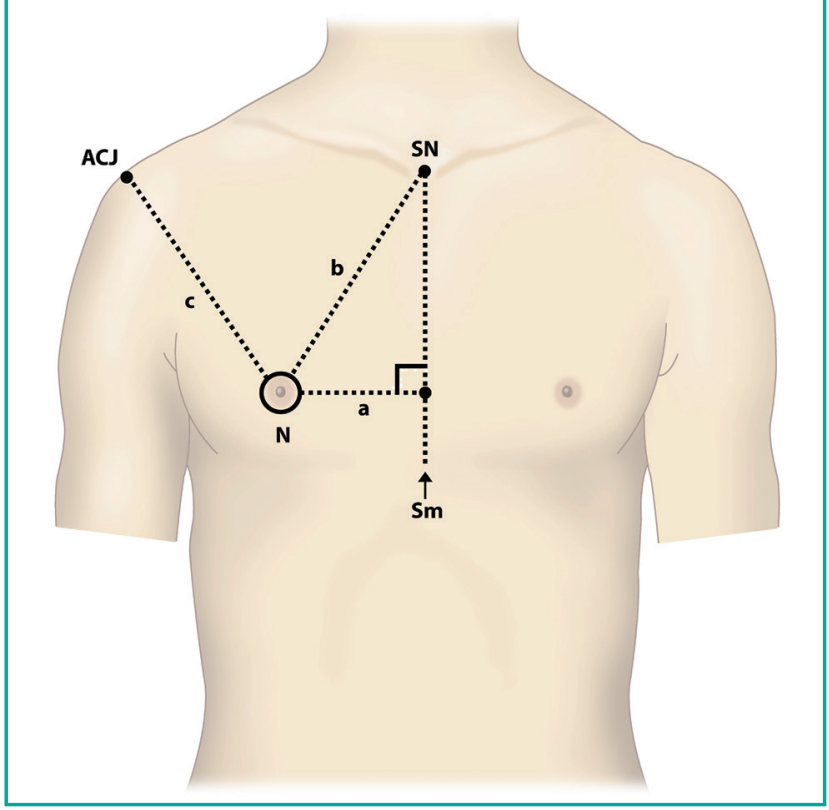

Comparisons between the groups were analyzed using SPSS ver. 6.1 (IBM, Somers, NY, USA). Numerical values were expressed as mean \pm standard deviation. The sternal midline-tonipple distance was designated as SMN; sternal notch-to-nipple distance as $\mathrm{SNN}$, and acromioclavicular joint-to-nipple distance as ACN (Fig. 1). Differences were considered statistically significant for $\mathrm{P}<0.05$ on regression analysis.

\section{Operation technique}

Tissue contours were evaluated while patients were sitting up in a chair prior to the operation. Excessively enlarged portions of the chest wall tissues marked along the border, and contour lines were used to differentiate the amount of tissue to be removed at various thoracic levels.

All patients received general anesthesia, and the breast tissue was accessed via a semi-circular incision along the bottom half of the areolar. To minimize the risk of NAC contracture, $1 \mathrm{~cm}$ thickness of breast tissue was left intact under the complex, while dissection remained superficial under the inferior skin flap. Staircase irregularity was avoided by using a dissection plane that extended from the skin flap to the inferior border of the pectoralis fascia Symmetry was respected as much as possible during the operation. In most patients, the mid-point between the lower part of the sixth rib and the second rib was used as the reference point, and the abnormally enlarged area to be incised was expanded or reduced. At this point, liposuction was used for further adjustment of the breast contour to the posteri- 
or axillary line. The NAC was secured to the underlying pectoralis fascia by one or two stitches. Drainage tubes were placed prior to closing the skin layers.

Mild compressive dressing was applied across the chest wall to reduce the risk of hematoma. Pressure to the area surrounding nipple and areola was minimized to prevent necrosis of the complex and skin flap. Skin tapes were applied to the inframammary folds for 2 weeks. Suction drainage was maintained until the daily output had decreased below $10 \mathrm{~mL}$. The stitches were removed 12 days after surgery.

\section{RESULTS}

In the patient group, 11 patients underwent subcutaneous mastectomy only, and the remaining 2 patients underwent subcutaneous mastectomy and liposuction. The average weight of excised breast tissue was $246 \mathrm{~g}$. No postoperative complications (hematoma, nipple necrosis, sensory deficit, asymmetry, underor overcorrection) were noted. Follow-up periods ranged from 6 to 19 months (mean, 9 months).

Mean ages were 19.8 years (range, 12 to 33 years) for the patient group and 25 years (range, 20 to 28 years) for the control group. The mean height and weight were $172.1 \mathrm{~cm}$ and $73.3 \mathrm{~kg}$ for the patient group and $175.7 \mathrm{~cm}$ and $68.1 \mathrm{~kg}$ for the control group.

In the patient group, the mean SMN were $125.9 \mathrm{~mm}$ and $110.3 \mathrm{~mm}$ for before and after operation measurements. The mean SNN were $214.7 \mathrm{~mm}$ and $188.2 \mathrm{~mm}$, respectively. The

\section{Table 1. Patient and control group data}

\begin{tabular}{|lccc|}
\hline Values & Patients & Controls & P-value \\
\hline Age $(\mathrm{yr})$ & $19.8 \pm 6.7$ & $22.3 \pm 1.2$ & 0.254 \\
Height $(\mathrm{cm})$ & $172.1 \pm 4.9$ & $175.7 \pm 6.2$ & 0.037 \\
Weight $(\mathrm{kg})$ & $73.3 \pm 16.0$ & $68.1 \pm 9.7$ & 0.018 \\
Body mass index $\left(\mathrm{kg} / \mathrm{m}^{2}\right)$ & 24.8 & 22.0 & 0.002 \\
\hline \multicolumn{4}{r}{} \\
\hline
\end{tabular}

Table 2. Mean anatomic parameters of patient and control groups

\begin{tabular}{|lccr|}
\hline Parameter & Preoperative & Postoperative & \multicolumn{1}{c|}{ Controls } \\
\hline Age (yr) & $19.8 \pm 6.7$ & $19.8 \pm 6.7$ & $22.3 \pm 1.2$ \\
Height (cm) & $172.1 \pm 4.9$ & $172.1 \pm 4.9$ & $175.7 \pm 6.2$ \\
Weight (kg) & $73.3 \pm 16.0$ & $73.3 \pm 16.0$ & $68.1 \pm 9.7$ \\
Diameter of nipple (cm) & $0.78 \pm 0.2$ & $0.7 \pm 0.2$ & $0.67 \pm 0.2$ \\
Sternal midline to nipple (cm) & $13.5 \pm 2.1$ & $12.1 \pm 1.4$ & $10.7 \pm 1.2$ \\
$\begin{array}{l}\text { Sternal notch to nipple (cm) } \\
\text { Sternal notch to xyphoid } \\
\text { process (cm) }\end{array}$ & $21.3 \pm 3.4$ & $16.8 \pm 1.7$ & $18.5 \pm 1.4$ \\
Acromioclavicular joint to & $20.9 \pm 3.4$ & $18.7 \pm 4.4$ & $22.4 \pm 2.1$ \\
$\quad$ nipple (cm) & $21.6 \pm 3.6$ & $21 \pm 3.8$ & $19.4 \pm 2.2$ \\
Circumference of thorax (cm) & $105 \pm 8.8$ & $95.9 \pm 7.8$ & $88.4 \pm 5.5$ \\
\hline \multicolumn{2}{l}{ Values are presented as mean \pm standard deviation. } \\
\hline
\end{tabular}

mean ACN were $209.0 \mathrm{~mm}$ and $187.8 \mathrm{~mm}$. In the control group, the same mean distances were $107.0 \mathrm{~mm}$ (SMN), 185.7 $\mathrm{mm}(\mathrm{SNN})$, and $214.0 \mathrm{~mm}(\mathrm{ACN})$ (Tables 1, 2). There was a statistical difference in the nipple location between the control group and the patient group $(\mathrm{P}<0.05)$. The comparisons are summarized in Tables 3 and 4.

A regression analysis was performed. Linear regression was defined as $y=a+b x ; y$ is a dependent variable representing the distance from the sternal notch to the nipple and the distance between the middle line of the sternum and the nipple; $x$ is an independent variable representing height and chest circumference; a is the intercept; and b is the slope or regression coefficient.

\section{The correlation between the height and the distance from the sternal notch to the nipple}

A scatter diagram and line of correlation between height and distance from the sternal notch to the nipple are presented graphically in Fig. 2. Here, h indicates height, and b, the distance from the sternal notch to the nipple.

Preoperative patient group: $\mathrm{b}=0.406 \mathrm{~h}-48.675(\mathrm{P}=0.079)$

Postoperative patient group: $\mathrm{b}=0.175 \mathrm{~h}-13.40(\mathrm{P}=0.129)$

Control group: $\mathrm{b}=0.122 \mathrm{~h}-2.921(\mathrm{P}=0.011)$

\section{The correlation between chest circumference and the distance from the sternal midline to the nipple}

A scatter diagram and line of correlation between chest circumference and distance from the sternal midline to the nipple are presented graphically in Fig. 3. Here, s indicates the average chest size, and a, the distance between the sternal midline and the nipple.

Preoperative patient group: $\mathrm{a}=0.213 \mathrm{~s}-8.884(\mathrm{P}=0.001)$

Postoperative patient group: $\mathrm{a}=0.125 \mathrm{~s}+0.075(\mathrm{P}=0.026)$

Control group: $\mathrm{a}=0.177 \mathrm{~s}-5.021(\mathrm{P}=0.001)$.

Table 3. Correlation between various parameters

\begin{tabular}{|llc|}
\hline Parameter & Correlated parameter & P-value \\
\hline Distance between nipples & Chest circumference & 0.001 \\
Sternal notch to nipple & Height & 0.001 \\
Sternal midline to nipple & Chest circumference & 0.001 \\
Distance between nipples & Sternal notch to nipple & 0.001 \\
\hline
\end{tabular}

Table 4. Correlation between preoperative, postoperative and control groups

\begin{tabular}{|llc|}
\hline Parameter & Correlated Parameter & P-value \\
\hline Distance between nipples & Chest circumference & 0.001 \\
Sternal notch to nipple & Height & 0.001 \\
Sternal midline to nipple & Chest circumference & 0.001 \\
Distance between nipples & Sternal notch to nipple & 0.001 \\
\hline
\end{tabular}




\section{Fig. 2. Correlation between $\mathrm{SN}-\mathrm{N}$, height}

Correlation between the distance of the sternal notch to the nipples (b) $(\mathrm{cm})$ and height $(\mathrm{cm})$ : preoperative (Pre op), postoperative (Post op), and control groups. SN, sternal notch; N, nipple.

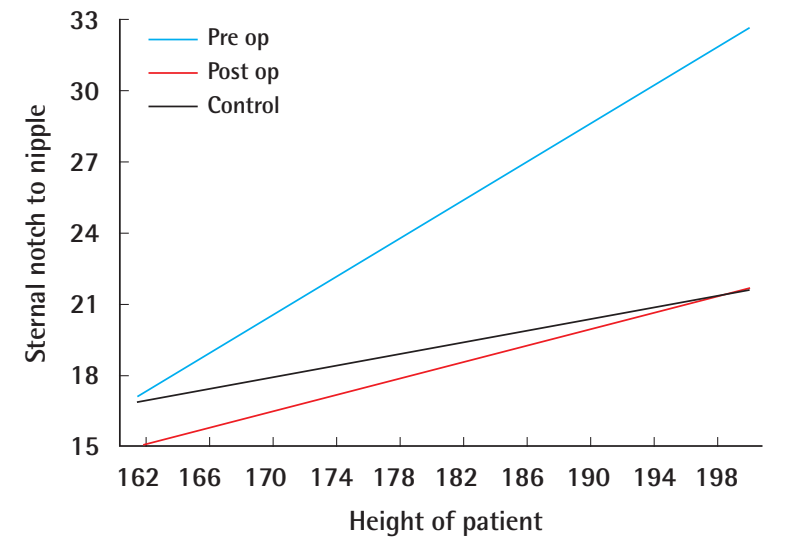

\section{Fig. 3. Corretlation between Sm-N, chest circumference}

Correlation between the distance of the sternal midline to the nipples (a) $(\mathrm{cm})$ and chest circumference $(\mathrm{cm})$ : preoperative (Pre op), postoperative (Post op), and control groups. Sm, sternal midline; $N_{1}$ nipple.

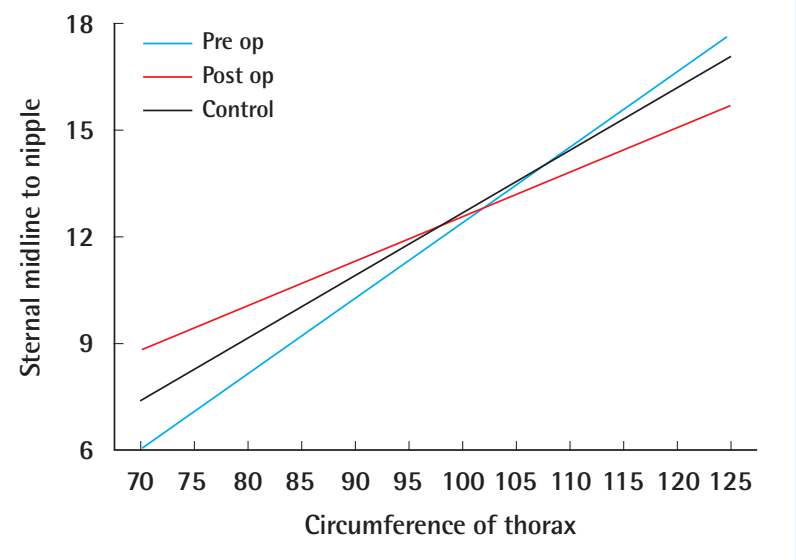

Fig. 4. A case of gynecomastia

A 14-year-old male with bilateral gynecomastia. (A) Preoperative photograph. (B) Appearance at 17 months after conventional subcutaneous mastectomy.
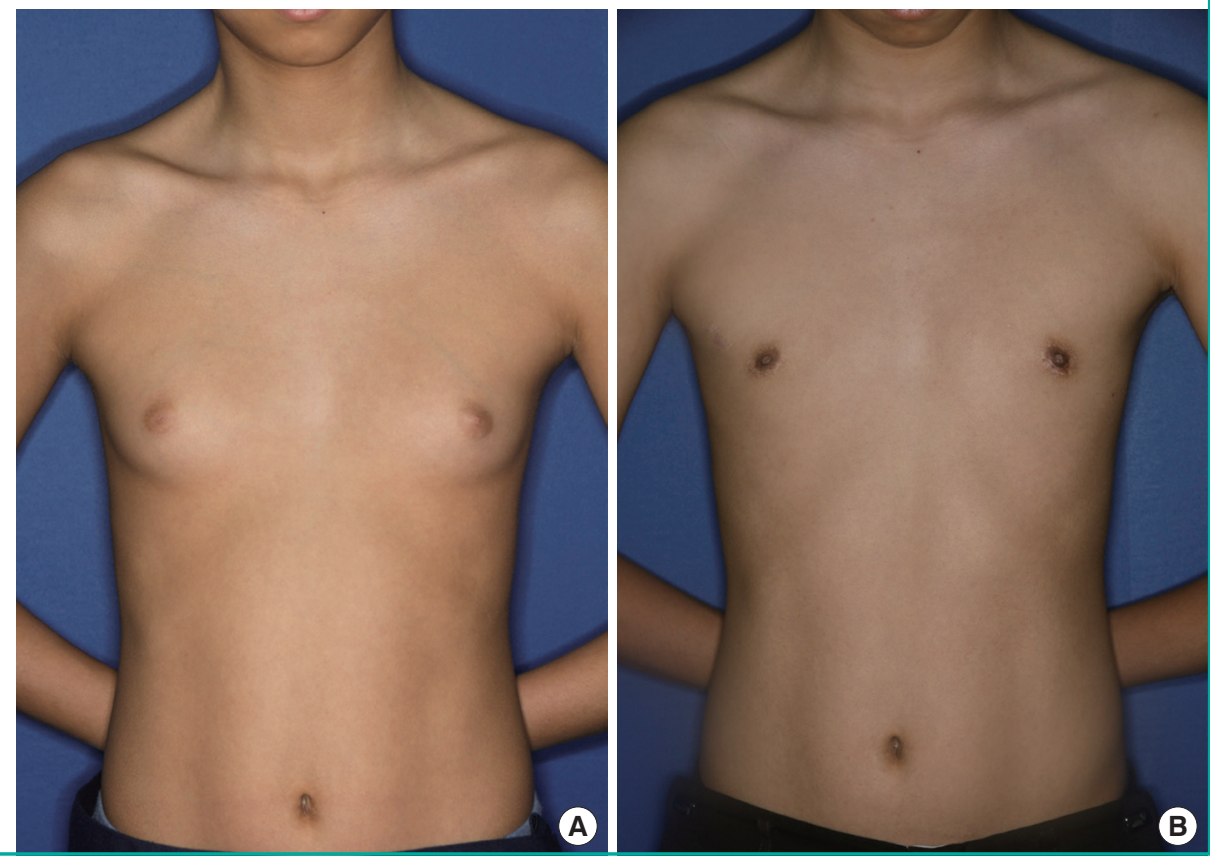

The post incisional scars were minimal; most patients did not complain about the scar itself. No contour irregularity was observed in postoperative chest wall. All of the patients evaluated the shape and location of NAC to be satisfactory. The mean satisfaction score was 4.3 (Fig. 4).

\section{DISCUSSION}

Gynecomastia can occur due to hypertrophy of the mammary gland (gynecomastia), fat accumulation (false gynecomastia), or complex proliferation. The condition should be treated upon clear understanding of the underlying cause [3]. Subcutaneous mastectomy is the primary method of breast excision. Liposuction is helpful for false gynecomastia, but is more helpful as a supplementary method of controlling contour to subcutaneous mastectomy.

Cordova and Moschella [4] proposed a morphological classification of gynecomastia (grade I-IV) and established a scale for choosing suitable operative techniques. Lanitis et al. [5] demonstated that any grade of gynecomastia can be corrected through circumaroelar incision, which gives better cosmetic results than other methods. Kasielska and Antoszewski [6] re- 
ported that subcutaneous mastectomy using a circumareolar incision without additional liposuction provides a good aesthetic outcome in patients with mild gynecomastia. All of our patients belong to a mild gynecomastia group (morphologic grades I and II), and subcutaneous mastectomy was sufficient for obtaining good aesthetic results.

In most cases, factors that affect the outcomes of gynecomastia surgery include reduction in breast protrusion, leveling of the nipple and areola, and new location for the nipple. Beckenstein et al. [7] had calculated the mean distances between sternal notch and nipple, between the middle line of sternum and nipple, and between the left and right nipples, and claimed that these values were correlated with height. However, we did not find that height correlated with any of the anthropometric data. Beer et al. [8] calculated a formula for the distance between middle line of sternum and nipple to extrapolate the optimal location for the NAC. Shulman et al. [9] verified the statistical relation between patient height, chest circumference, the height of nipples, and distance from sternal notch to each nipple. They observed a statistical correlation between chest circumference and distance between the nipples. This study also used the method as described by Beer et al. [8] and Shulman et al. [9], but given that the chest circumference of Koreans is smaller than that of westerners, some variables had to be adjusted accordingly.

In female patients with breast hypertrophy, simply fixing the NAC to the surrounding skin was not adequate. Over a longterm, the nipple was shown to descend unless fixed to the underlying subcutaneous tissue, which highlights the need for an objective evaluation of post-surgical nipple location in male patients. Among the 13 patients in our study, the post-surgical nipple location was assessed using the same anatomical reference points used for preoperative assessment. We found that the bestfit equation between patient height and SNN distance to be $b=0.406 h-48.675(P=0.079)$ before surgery and $b=0.175 h-$ $3.40(\mathrm{P}=0.129)$ after surgery. This compares to $\mathrm{b}=0.122 \mathrm{~h}-$ $2.921(\mathrm{P}=0.011)$ in the control group. For the correlation between the chest circumference and the distance between the mid-line of the sternum and the nipple, the best-fit equation was $\mathrm{a}=0.213 \mathrm{~s}-8.884(\mathrm{P}=0.001)$ before surgery and $\mathrm{a}=0.125 \mathrm{~s}+$ $0.075(\mathrm{P}=0.026)$ after surgery. For the control group, the equation was $\mathrm{a}=0.177 \mathrm{~s}-5.021(\mathrm{P}=0.001)$. Comparing the difference in the location of the center of nipple, the slopes for the heightdistance from the sternal notch to the nipple and chest circumference-distance between the mid-line of the sternum and the nipple were 0.406 and 0.213 preoperatively and 0.175 and 0.125 postoperatively, respectively. The slopes of the control group were 0.122 and 0.177 , respectively, showing that when the slope was compared in cases of conventional surgery, the postoperative slope and the slope in the control group were statistically significantly different. These findings indicate that preoperative nipple locations were more inferolateral in gynecomastia patients when compared to controls, and the operations were associated with bringing the nipple closer to normal values. However, the sample size of the patient group was not large enough to estimate the degree of sagging relative to the amount of breast tissue.

Nipple locations were compared between gynecomastia patients undergoing subcutaneous mastectomy and control subjects. Nipple positions were considerably lower in patients with gynecomastia than in control subjects. Subcutaneous mastectomy was associated with mild elevations, but postoperative locations were still lower compared to controls. Further efforts are needed to improve the location of postoperative NAC in patients with gynecomastia.

\section{REFERENCES}

1. Mageed MA. Surgical treatment for moderate and largesized gynecomastia. Egypt J Plast Reconstr Surg 2007;31: 45-55.

2. Park JH, Lee YH. The modified surgical treatment of gynecomastia: pan-cake method. J Korean Soc Plast Reconstr Surg 2007;34:628-34.

3. Kim J, Kim IG, Uhm KI, et al. Clinical analysis of the gynecomastia. J Korean Soc Plast Reconstr Surg 1991;18:1131-7.

4. Cordova A, Moschella F. Algorithm for clinical evaluation and surgical treatment of gynaecomastia. J Plast Reconstr Aesthet Surg 2008;61:41-9.

5. Lanitis S, Starren E, Read J, et al. Surgical management of Gynaecomastia: outcomes from our experience. Breast 2008; 17:596-603.

6. Kasielska A, Antoszewski B. Surgical management of gynecomastia: an outcome analysis. Ann Plast Surg 2013;71: 471-5.

7. Beckenstein MS, Windle BH, Stroup RT Jr. Anatomical parameters for nipple position and areolar diameter in males. Ann Plast Surg 1996;36:33-6.

8. Beer GM, Budi S, Seifert B, et al. Configuration and localization of the nipple-areola complex in men. Plast Reconstr Surg 2001; 108:1947-52.

9. Shulman O, Badani E, Wolf Y, et al. Appropriate location of the nipple-areola complex in males. Plast Reconstr Surg 2001;108:348-51. 